\title{
Design of a self-unfolding delivery concept for oral administration of macromolecules
}

Jørgensen, Jacob R.; Thamdrup, Lasse H.E.; Kamguyan, Khorshid; Nielsen, Line H.; Nielsen, Hanne M.; Boisen, Anja; Rades, Thomas; Müllertz, Anette

Published in:

Journal of controlled release

Link to article, DOI:

10.1016/j.jconrel.2020.10.024

Publication date:

2021

Document Version

Peer reviewed version

Link back to DTU Orbit

Citation $(A P A)$ :

Jørgensen, J. R., Thamdrup, L. H. E., Kamguyan, K., Nielsen, L. H., Nielsen, H. M., Boisen, A., Rades, T., \& Müllertz, A. (2021). Design of a self-unfolding delivery concept for oral administration of macromolecules. Journal of controlled release, 329, 948-954. https://doi.org/10.1016/j.jconrel.2020.10.024

\section{General rights}

Copyright and moral rights for the publications made accessible in the public portal are retained by the authors and/or other copyright owners and it is a condition of accessing publications that users recognise and abide by the legal requirements associated with these rights.

- Users may download and print one copy of any publication from the public portal for the purpose of private study or research.

- You may not further distribute the material or use it for any profit-making activity or commercial gain

- You may freely distribute the URL identifying the publication in the public portal 


\section{Design of a self-unfolding delivery concept for oral administration of macromolecules}

Jacob R. Jørgensen ${ }^{a}$, Lasse H. E. Thamdrup ${ }^{b}, K_{\text {Khorshid Kamguyan }}^{b}$, Line H. Nielsen ${ }^{b}$, Hanne M. Nielsen ${ }^{c}$, Anja Boisen ${ }^{b}$, Thomas Rades ${ }^{a}$, Anette Müllertz ${ }^{d, *}$

${ }^{a}$ Department of Pharmacy, University of Copenhagen, Universitetsparken 2, 2100 Copenhagen, Denmark

E-mail addresses: jacob.r.joergensen@sund.ku.dk, thomas.rades@sund.ku.dk

${ }^{\mathrm{b}}$ Center for Intelligent Drug Delivery and Sensing Using Microcontainers and Nanomechanics (IDUN), Department of Health Technology, Technical University of Denmark, Ørsteds Plads, 2800 Kgs. Lyngby, Denmark

E-mail addresses: Ihth@dtu.dk, khokam@dtu.dk, lihan@dtu.dk, aboi@dtu.dk

${ }^{\mathrm{c}}$ Center for Biopharmaceuticals and Biobarriers in Drug Delivery, Department of Pharmacy, University of Copenhagen, Universitetsparken 2, 2100 Copenhagen, Denmark E-mail address: hanne.morck@sund.ku.dk

${ }^{\mathrm{d}}$ Bioneer:FARMA, Department of Pharmacy, University of Copenhagen, Universitetsparken 2, 2100 Copenhagen, Denmark

* Corresponding author at: Universitetsparken 2, 2100 Copenhagen, Denmark E-mail address: anette.mullertz@sund.ku.dk (A. Müllertz)

Keywords: Oral insulin; Delivery devices; Elastomers; Permeation enhancers; Protease inhibitors; Polydimethylsiloxane

\section{Abstract}

Delivering macromolecular drugs, e.g. peptides, to the systemic circulation by oral administration is challenging due to their degradation in the gastrointestinal tract and low transmucosal permeation. In this study, the concept of an oral delivery device utilizing an elastomeric material is presented with the potential of increasing the absorption of peptides, e.g. insulin. Absorption enhancement in the intestine is proposed as a result of self-unfolding of a polydimethylsiloxane foil upon release from enteric coated capsules. A $\mathrm{pH}$-sensitive polymer coating prevents capsule disintegration until arrival in the small intestine where complete unfolding of the elastomeric foil ensures close contact with the intestinal mucosa. Foils with close-packed hexagonal compartments for optimal drug loading are produced by casting against a deep-etched silicon master. Complete unfolding of the foil upon capsule disintegration is verified in vitro and the insulin release profile of the final delivery device confirms insulin protection at gastric $\mathrm{pH}$. In vivo performance is evaluated with the outcome of quantifiable plasma insulin concentrations in all rats receiving duodenal administration of the novel delivery device. By taking advantage of elastomeric material properties for drug delivery, this approach might serve as inspiration 
44 for further development of commercially viable biocompatible devices for oral delivery of 45 macromolecules.

\section{Introduction}

47 Oral administration of macromolecules, e.g. peptides and proteins, has been attempted for decades in order to improve patient compliance [1,2]. Previous initiatives have mainly focused on incorporating protease inhibitors, such as soybean trypsin inhibitor (STI), and permeation enhancers (PEs) in order to decrease enzymatic degradation while increasing absorption from the intestine [1,3]. Co-formulating peptides with such active excipients in tablets or capsules has been the strategy in several preclinical and clinical assessments [2]. However, innovative oral delivery devices have, over the last few years, challenged the conventional concepts of oral systemic absorption by proposing the application of gastrointestinal (Gl) injections resulting in $>10 \%$ oral bioavailability relative to subcutaneous (SC) injection [4-7]. Physical perforation of the outer layers of the absorptive barriers has thereby become an interesting alternative to excipient-induced absorption of macromolecules [8]. While both approaches are interesting platforms for peptide delivery, the risks of repeated administrations of PEs and GI-injections are unknown. Nevertheless, the constant regeneration of the epithelial cells along the Gl-tract is a recurring argument for the therapeutic relevance of temporary barrier disruption regardless of being induced by PEs or by physical perforation $[5,9,10]$.

63 The status as food additive, Generally Recognized As Safe (GRAS) or inactive ingredient held by some PEs, e.g. sodium caprate $\left(\mathrm{C}_{10}\right)$, salcaprozate sodium (SNAC) and sodium dodecyl sulfate (SDS), have rendered them popular choices in industrial formulation strategies [8]. Notably, $\mathrm{C}_{10}$ and SNAC have proven beneficial, and have led to the achievement of therapeutic oral bioavailability of insulin and the glucagon-like peptide-1 agonist, semaglutide, respectively. Moreover, the latter formulation has received approval from the U.S. Food \& Drug Administration [11,12]. Thereby, PE-based formulations have proven to be commercially viable, yet they still need to compensate with higher peptide doses due to relatively low oral bioavailability compared to the Gl-injecting delivery devices $[5,7,11,12]$. On the other hand, while the Gl-injecting devices are innovative from an engineering perspective, their complexity is likely to impede the rate of potential mass production compared to that of conventional tablets and capsules [4,5,7]. A solution to the high doses required in PE-based oral dosage forms has previously been proposed with unidirectional-releasing devices capable of creating local environments of high 
concentrations of peptide and excipients along the intestinal absorptive barrier [13-16]. Such confinement would increase the local effect of active excipients, e.g. PEs and protease inhibitors, while creating a steep peptide concentration gradient across the intestinal mucosa. The proximity between the absorptive barrier and such unidirectionalreleasing devices has proven of outmost importance in vitro with a 50\% decrease in insulin absorption for every distance increase of $130 \mu \mathrm{m}$ from a Caco-2 cell monolayer mimicking the intestinal absorptive barrier [16].

Insulin release in the gastric environment can be prevented by enteric coating, but ensuring optimal unidirectional release in close proximity to the intestinal epithelium has proven challenging to attain in vivo [17]. Moreover, it has proven problematic to conduct in vivo studies of enteric coated capsules in rats, due to long-term gastric retention of such dosage forms [18]. Previously, this issue has been circumvented by pre-administration of the prokinetic agent, metoclopramide, to promote gastric emptying, or by direct intestinal insertion of the dosage form through an incision in the proximal small intestine [13,14,19]. Thus, such preclinical in vivo studies either alter the normal peristalsis by inclusion of a prokinetic agent or might even possess the risk of absorption at the site of intestinal perforation, thereby causing a false positive result.

An alternative approach to preclinical in vivo studies of enteric coated capsules in rats was used in the present work, without neither penetrating the Gl-tract mucosa nor using prokinetic agents. This in vivo approach, which will be discussed in more detail below, contributed to the overall aim of the present study, namely to conceptualize and design an oral device capable of guaranteeing optimal unidirectional drug and excipient release in close proximity to the intestinal mucosa. The design comprises an elastomeric selfunfolding foil with cavities for drug- and excipient loading rolled up in an enteric coated gelatin capsule. The key to successfully achieving unidirectional release in close proximity to the intestinal mucosa thus lies in the elastomeric nature of the material ensuring purely elastic deformation. For the present proof-of-concept study, polydimethylsiloxane (PDMS) was chosen as the foil material due to its elastomeric properties, while the approximately 6 kDa peptide hormone, insulin, was chosen as the model macromolecular drug. 


\subsection{Materials}

108

109

110

111

112

113

114

115

116

117

Human recombinant insulin, SDS and dibutyl sebacate were acquired from Sigma-Aldrich (St. Louis, MO, USA). STI was bought from Thermo Fisher Scientific (Waltham, MA, USA) and SYLGARD ${ }^{\text {TM }} 184$ silicone elastomer kit from Dow Chemical Company (Midland, MI, USA). Eudragit ${ }^{\circledR}$ L 100 and L100-55 were both obtained from Evonik (Essen, Germany). Midazolam (5 mg mL ${ }^{-1}$ ) was procured from Hameln (Gloucester, UK) whereas Hypnorm (fentanyl, $0.315 \mathrm{mg} \mathrm{mL}^{-1}$; fluanisone $10 \mathrm{mg} \mathrm{mL}^{-1}$ ) was acquired from Skanderborg Pharmacy (Skanderborg, Denmark), and pentobarbital/Euthanimal (400 mg mL $\mathrm{m}^{-1}$ ) from Alfasan (Woerden, Netherlands). All additional chemicals and solvents were at least of analytical grade and obtained from commercial suppliers. Ultrapure water was used throughout the studies purified by an Ultra Clear UV system (Evoqua Water Technologies, Pittsburgh, PA, USA).

\subsection{Fabrication of hexagonal patterned silicon master}

For optimal results during the deep anisotropic etch into the silicon ( $\mathrm{Si}$ ) substrate, a $\mathrm{SiO}_{2}$ hard mask was used. A $1 \mu \mathrm{m}$ thick wet thermal oxide was grown in a horizontal furnace (Tempress, Vaassen, The Netherlands) and masked using the conventional positive photoresist $A Z^{\circledR} 5214 \mathrm{E}$ (MicroChemicals, Ulm, Germany). In order to increase resist adhesion, hexamethyldisilizane was deposited as part of the procedure for spin coating the $1.5 \mu \mathrm{m}$ thick resist layer. The UV-exposure was conducted using an MLA100 Tabletop Maskless Aligner (Heidelberg Instruments, Heidelberg, Germany) and a dose of $90 \mathrm{~mJ} \mathrm{~cm}^{-}$ ${ }^{2}$ before developing the exposed pattern for $90 \mathrm{~s}$ using $A Z^{\circledR} 726$ MIF (MicroChemicals, Ulm, Germany). Subsequently, the hexagonal pattern was transferred into the oxide layer using an Advanced Oxide Etcher (STS MESC Multiplex ICP, SPTS Technologies, Newport, UK) with $\mathrm{C}_{4} \mathrm{~F}_{8}$ and $\mathrm{H}_{2}$ as the reactive gasses. The remaining resist mask was stripped using a combination of energetic oxygen plasma in a barrel asher (300 Semi Auto Plasma Processor, PVA TePla, Wettenberg, Germany) and submersion into concentrated $\mathrm{H}_{2} \mathrm{SO}_{4}$ with $\left(\mathrm{NH}_{4}\right)_{2} \mathrm{~S}_{2} \mathrm{O}_{8}$ salt at $80^{\circ} \mathrm{C}$. The honeycomb trenches were then etched into the Si using an inductively coupled plasma deep reactive ion etching tool (STS Pegasus, SPTS Technologies, Newport, UK). The tool utilizes a Bosch-type process for performing deep anisotropic etching of $\mathrm{Si}$ by alternating between $\mathrm{Si}$ etching using $\mathrm{SF}_{6}$ and $\mathrm{O}_{2}$ and sidewall passivation obtained by deposition of $\mathrm{C}_{4} \mathrm{~F}_{8}$. The substrate temperature was kept 
at $0{ }^{\circ} \mathrm{C}$ throughout the etching process and the substrate was cleaned using oxygen plasma and a mixture of concentrated $\mathrm{H}_{2} \mathrm{SO}_{4}$ and $\mathrm{H}_{2} \mathrm{O}_{2}(4: 1 \mathrm{v} / \mathrm{v}$, commonly referred to as piranha) before stripping the remaining oxide mask in aqueous buffered hydrofluoric acid $(12 \%, v / v)$ with $\mathrm{NH}_{4} \mathrm{~F}$. As the Si master was intended for foil fabrication by means of casting with PDMS, an anti-stick coating was deposited by molecular vapor deposition (MVD 100 Molecular Vapor Deposition System, Applied Microstructures, Orbotech, Yavne, Israel). This effectively created a monolayer of $1 \mathrm{H}, 1 \mathrm{H}, 2 \mathrm{H}, 2 \mathrm{H}$-perflourodecyltrichlorosilane, which decreased the surface energy of the Si master. This in turn promoted demolding of the delicate honeycomb protrusions after the PDMS casting and ensured that elastomer rip-off was prevented, thereby preserving the pristine master for multiple replication cycles. The width and depth of the hexagonal trenches were measured by vertical scanning interferometry using a PLu Neox 3D Optical Profiler (Sensofar Metrology, Terrassa, Spain).

\subsection{Fabrication of PDMS foil}

The self-unfolding elastomer foils were fabricated by casting with SYLGARD ${ }^{\mathrm{TM}} 184$, which is a two-component product consisting of a PDMS base and a curing agent. The base resin and curing agent were mixed in a 10:1 ratio $(\mathrm{w} / \mathrm{w})$ and degassed in a desiccator for 30 min prior to use. The mixture was then poured onto the silicon master and degassed in a desiccator once more in order to remove potential air bubbles, which would otherwise compromise the structure replication fidelity. The uncured PDMS replica was then kept in an oven at $37^{\circ} \mathrm{C}$ overnight and the cured elastomer foil was subsequently peeled from the Si master. The topography of the foils was characterized using vertical scanning interferometry on a PLu Neox 3D Optical Profiler (Sensofar Metrology, Terrassa, Spain). In vitro assessment of the self-unfolding properties of the foil was assessed by recording the unfolding of a PDMS foil $\left(7 \times 7 \mathrm{~mm}^{2}\right)$ as a result of capsule disintegration in water at $37{ }^{\circ} \mathrm{C}$ using a Dino-Lite Premier AM7013MZT digital microscope (AnMo Electronics Corporation, New Taipei City, Taiwan).

\subsection{Preparation of the final oral delivery device}

PDMS foils were loaded with a powder mixture of insulin, SDS as PE and STI (5:3:2, $\mathrm{w} / \mathrm{w} / \mathrm{w}$ ) by tapping the powder on top of the foil and gently scraping off any excess amount. A solution of Eudragit ${ }^{\circledR} \mathrm{L} 100(1 \%, \mathrm{w} / \mathrm{v})$ and dibutyl sebacate $(0.1 \%, \mathrm{w} / \mathrm{v})$ in isopropanol (IPA) was then spray coated on top of the hexagonal openings to seal the powder inside 
170 thus enabling subsequent handling of the foils. An ExactaCoat Ultrasonic Spray System

171 (Sono-Tek, Milton, NY, USA) was used for this purpose with an infuse rate of $0.05 \mathrm{~mL} \mathrm{~min}$

$172{ }^{1}$, path speed of $5 \mathrm{~mm} \mathrm{~s}^{-1}$ and shaping air pressure of 0.02 mbar. The generator power

173 was set to $2.2 \mathrm{~W}$ and an AccuMist nozzle was applied with a sample-to-nozzle distance of

$17450 \mathrm{~mm}$. Immediately after spray coating, the foils were gently rolled between the finger tips

175 and inserted into size 9 gelatin capsules (Torpac, Fairfield, NJ, USA). A flat Si-chip was

176 additionally coated to determine the coating thickness of Eudragit ${ }^{\circledR} \mathrm{L} 100$ by scanning

177 electron microscopy using a Hitachi TM3030 Plus tabletop microscope (Hitachi High-

178 Technologies Europe, Krefeld, Germany) with an accelerating voltage of $15 \mathrm{kV}$. Nine

179 delivery devices were prepared in total out of which three were used for testing for the

180 uniformity of insulin content, three were used for in vitro insulin release studies and three

181 prepared for in vivo insulin absorption studies. The gelatin capsules for in vivo studies

182 were additionally loaded with a cylindrical power magnet with a diameter and height of $2 \times$

$1831 \mathrm{~mm}$, respectively (Magnetz og Magnordic, Hvidovre, Denmark). Enteric coating of the

184 gelatin capsules was carried out by two dip coating cycles, first dipping the capsule body

185 and afterwards the cap in Eudragit ${ }^{\circledR} \mathrm{L} 100-55$ (15\%, w/v) and dibutyl sebacate $(0.75 \%$,

$186 \mathrm{w} / \mathrm{v}$ ) in IPA giving the final delivery device. Images of the delivery device were captured

187 with a Dino-Lite Premier AM7013MZT digital microscope (AnMo Electronics Corporation,

188 New Taipei City, Taiwan).

\subsection{In vitro release of insulin}

190 A test for uniformity of insulin content was initially carried out by fully dissolving the content 191 of three delivery devices in $10 \mathrm{~mL}$ of $50 \mathrm{~mm}$ phosphate buffer at $\mathrm{pH} 7$ followed by reversed 192 phase high-performance liquid chromatography (HPLC) as described below. In vitro 193 release studies were carried out on a 400-DS Apparatus 7 (Agilent Technologies, Santa 194 Clara, CA, USA) equipped with $5 \mathrm{~mL}$ sample cells and supplied with a $50 \mathrm{~mm}$ citrate buffer 195 at $\mathrm{pH} 4$ and a $50 \mathrm{~mm}$ phosphate buffer at $\mathrm{pH}$ 7. The oral delivery device was placed in 50 196 mesh basket sample holder and dissolution carried out under sink conditions at $37^{\circ} \mathrm{C}$ for 1 197 hour at pH 4 followed by 5 hours at pH 7 with a dip speed of 15 dips per minute. Automatic 198 sampling of $0.5 \mathrm{~mL}$ was timed at $10,30,60,70,80,90,105,120,150,180,240$ and 360 $199 \mathrm{~min}$, each replaced with fresh dissolution medium. After sampling at $60 \mathrm{~min}$, the total 200 volume of citrate buffer in the sample cell was fully replaced with phosphate buffer. Insulin 201 quantification was immediately carried out by HPLC on a Dionex Ultimate 3000 system 202 (Thermo Fisher Scientific, Waltham, MA, USA) equipped with a Kinetex XB-C18 column $203(100 \times 4.6 \mathrm{~mm}, 5 \mu \mathrm{m}, 100 \AA$; Phenomenex, Torrance, CA, USA). The following gradient of 
mobile phase A: $0.1 \%(\mathrm{v} / \mathrm{v})$ trifluoroacetic acid (TFA) in water and $\mathrm{B}: 0.1 \%(\mathrm{v} / \mathrm{v})$ TFA in acetonitrile was applied with a flow rate of $0.5 \mathrm{~mL} \mathrm{~min}^{-1}: 0-6.0 \mathrm{~min} A: B(77: 23$ to 50:50, $\mathrm{v} / \mathrm{v}), 6.0-6.5 \mathrm{~min} A: B(50: 50$ to $77: 23, \mathrm{v} / \mathrm{v})$ and $6.5-8.0 \mathrm{~min} A: B(77: 23, \mathrm{v} / \mathrm{v})$. Insulin was quantified as the area under the curve of the UV-absorbance peak at $276 \mathrm{~nm}$ against a standard curve from $10-1000 \mu \mathrm{g} \mathrm{mL}^{-1}$ with an injection volume of $20 \mu \mathrm{L}$ and column temperature of $22^{\circ} \mathrm{C}$.

\subsection{In vivo absorption of insulin}

The animal experiments were carried out in accordance with the Danish law on animal experiments as approved by the Danish Animal Experiments Inspectorate in concordance with the EU directive 2010/63/EU under license no. 2016-15-0201-00892. In total, 11 male Sprague Dawley rats (Janvier Labs, Le Genest-Saint-Isle, France) weighing 255 - $310 \mathrm{~g}$ were divided into three groups and housed with reversed day/night rhythm (12/12 h). The rats were fasted with ad libitum access to water for $12-14 \mathrm{~h}$ before initiating the study. Bolus anesthesia of fentanyl (236 $\left.\mathrm{g} \mathrm{kg} \mathrm{k}^{-1}\right)$, fluanisone $\left(7.5 \mathrm{mg} \mathrm{kg}^{-1}\right)$ and midazolam (3.75 $\mathrm{mg} \mathrm{kg}^{-1}$ ) was given as SC injections with repeated SC injections every 30 min of $1 / 3$ of the bolus dose together with $0.4 \mathrm{~mL}$ saline. The abdominal cavity was opened to expose the gastrointestinal tract prior to dosing of all 11 rats. Two groups, each of three rats, were administered SC injections of insulin $\left(1 \mathrm{IU} \mathrm{kg}^{-1}\right)$ or saline as positive and negative control, respectively. Five rats were administered the novel delivery device directly to the stomach by the use of an oral gavage dosing tube. Gastric emptying was then facilitated by dragging the delivery device into the duodenum about $3 \mathrm{~cm}$ from the pylorus with an external magnet $(\mathrm{t}=0 \mathrm{~min})$. Blood samples of $200 \mu \mathrm{L}$ were taken from the tail vein into Microvette ${ }^{\circledR} 200$ K3E tubes (Sarstedt, Nümbrecht, Germany) at 10, 20, 30, 45, 60, 90, 120 and $180 \mathrm{~min}$ followed by isolation of the plasma by centrifugation at $9,300 \times \mathrm{g}$ at $4{ }^{\circ} \mathrm{C}$ using a Microcentrifuge 5415 R (Eppendorf, Hamburg, Germany). Plasma samples were stored at $-20^{\circ} \mathrm{C}$ until insulin quantification was carried out by an enzyme-linked immunosorbent assay (ELISA) as described by the manufacturer (Mercodia, Uppsala, Sweden). The rats were euthanized by intracardiac injections of pentobarbital (100 mg kg $\left.{ }^{1}\right)$ after the final blood sampling. Additionally, diluted in vitro release samples were analyzed by ELISA in order to confirm preservation of the antibody-binding moieties of insulin upon release from the prepared delivery device.

\subsection{Data treatment}


236 All data were treated using Microsoft Excel 2010 (Redmond, WA, USA) and GraphPad

237 Prism version 8.3.0 (San Diego, CA, USA) and expressed as mean \pm standard deviation 238 (SD) unless stated otherwise.

\section{Results and discussion}

\subsection{Preparation and in vitro assessment of the foil-based delivery device}

241 An overview of the preparation steps of the drug loaded elastomeric foil is shown in Fig.

$2421 \mathrm{~A}$, and the principle of its proposed absorption enhancing mechanism illustrated in Fig.

$2431 \mathrm{~B}$.

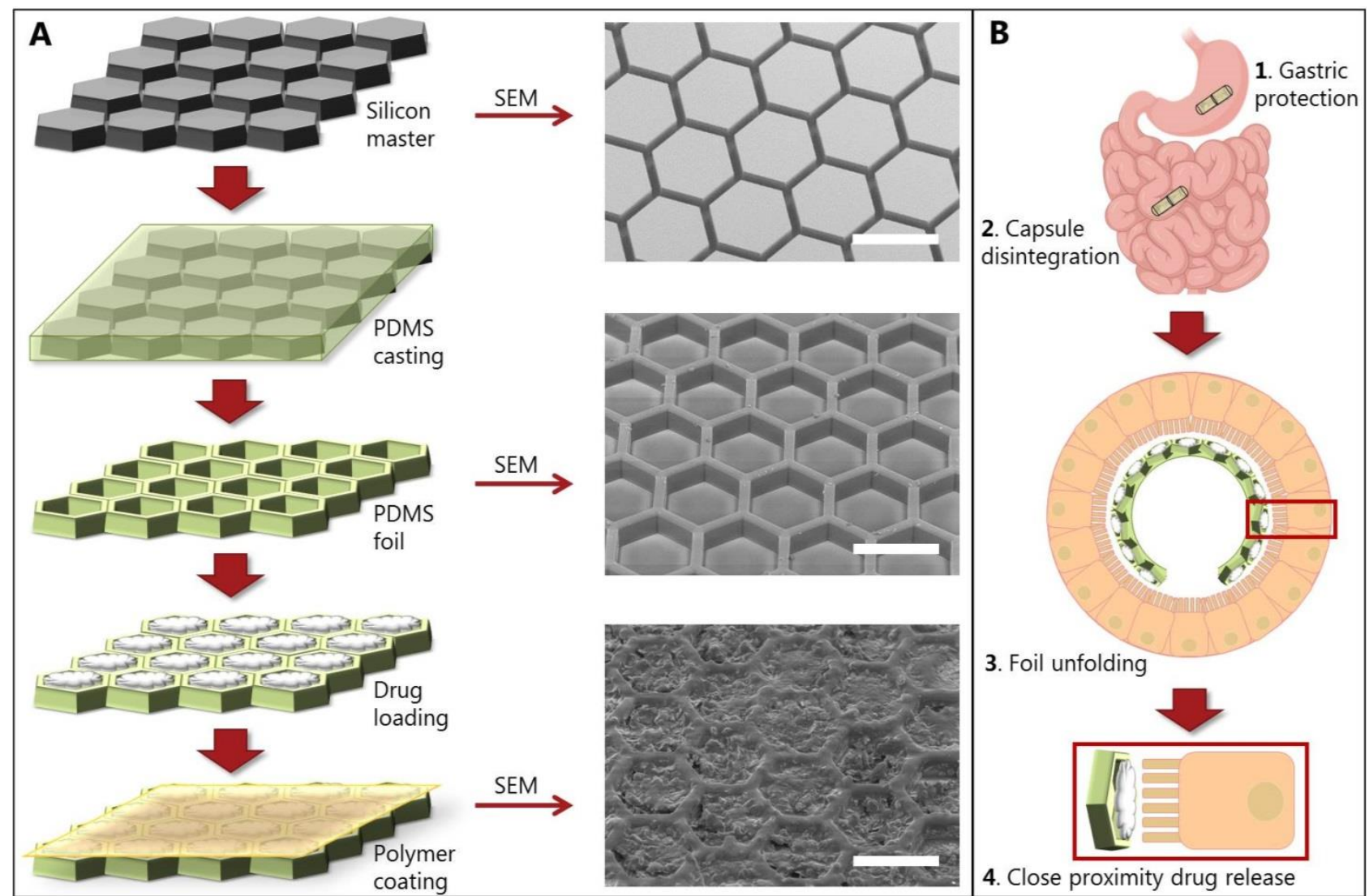

Fig. 1. Illustrations of the preparation steps and the principle of the delivery device (A) Fabrication and preparation steps of drug-loaded polydimethylsiloxane (PDMS) foil with scanning electron microscopy (SEM) images, scale bars: $400 \mu \mathrm{m}$ (B) Principle of the oral delivery device: Intestinal capsule disintegration is followed by foil unfolding and unidirectional drug release in close proximity to the absorptive barrier (the gastrointestinal tract schematic was created with Biorender.com) - (2column fitting image).

251 An early $1^{\text {st }}$ generation design of the cavities for drug- and excipient loading in the PDMS 252 foil was based on previously published cylindrical devices $[20,21]$. This $1^{\text {st }}$ generation foil 
designed with cylindrical cavities was used for initial in vitro assessment of the selfunfolding properties of the PDMS foil (Fig. 2, Video S1).
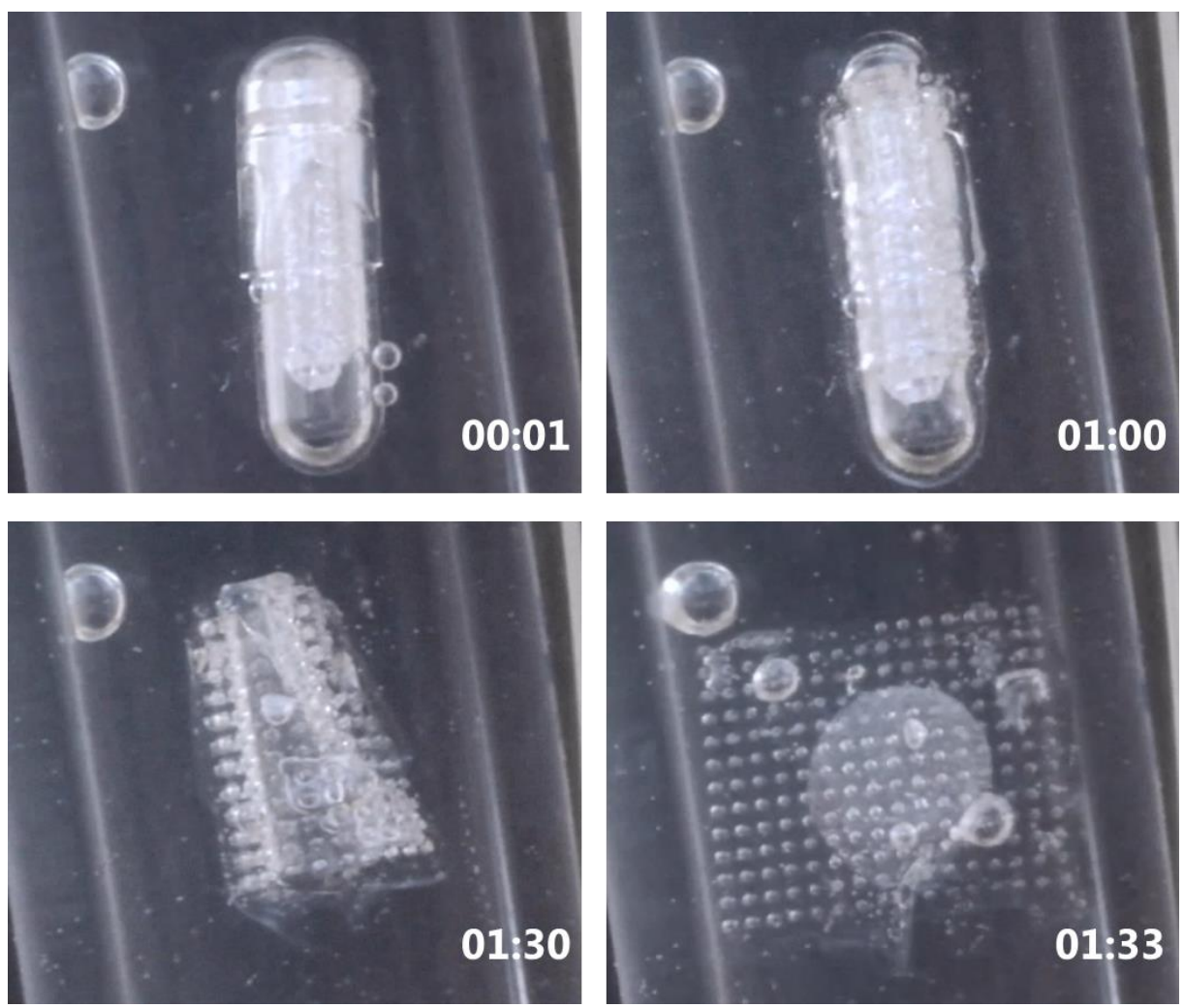

Fig. 2. Real-time disintegration of a size 9 gelatin capsule in water at $37^{\circ} \mathrm{C}$ following unfolding of a PDMS foil with cylindrical cavities, timer shown as MM:SS - (1.5-column fitting image).

The confined shape of the foil in the gelatin capsule proved completely reversible with the foil fully restoring its original flat shape upon capsule disintegration. Inside the tubular confinement of the rat duodenum, measuring about $2.5-3 \mathrm{~mm}$ in diameter, such unfolding would thus result in close proximity between the drug loaded cavities of the foil and the intestinal mucosa [22]. To further enhance the chances of oral insulin absorption, the Si master with a close-packed hexagonal tiling was deep-etched and used for fabrication of the $2^{\text {nd }}$ generation foil (Fig. 1A). The width and depth of the hexagonal trenches of the Si master were $46 \pm 2.2$ and $127 \pm 0.8 \mu$ m (mean \pm SD), $n=5$ ), respectively (Fig. S1A). Subsequent PDMS casting resulted in thin foils with a welldefined surface topography composed of protrusions arranged in a honeycomb pattern with an average height of $125 \pm 0.6 \mu \mathrm{m}$ (mean \pm SD, $n=5$ ), (Fig. S1B). The hexagonal design of the $2^{\text {nd }}$ generation foil resulted in a functional area for drug- and excipient loading of $78 \%$ compared to only $21 \%$ for the $1^{\text {st }}$ generation foil with cylindrical cavities. The elastomeric PDMS foil with hexagonal cavities was cut into pieces of $7 \times 7 \mathrm{~mm}^{2}$, and loaded with a powder mixture consisting of insulin, SDS and STI. A covering layer of 
273 Eudragit $^{\circledR} \mathrm{L} 100$, soluble in intestinal fluids with $\mathrm{pH}>6$, was applied by spray coating to 274 seal the powder mixture inside the cavities. The covering layer, measuring approximately $27519 \mu \mathrm{m}$ (Fig. S2), thus ensured that no loss of loaded powder occurred when rolling up and inserting the foil into size 9 gelatin capsules. Finally, dip coating of the gelatin capsules was performed using Eudragit ${ }^{\circledR} \mathrm{L} 100-55$ to allow for rapid capsule disintegration when reaching a pH value above 5.5 in the rat small intestine. The uniformity of insulin content in the delivery devices was quantified as $606 \pm 65 \mu \mathrm{g}$ corresponding to $17.5 \pm 1.9$ international units (IU), when loading a powder mixture of insulin, SDS and STI (5:3:2 $\mathrm{w} / \mathrm{w} / \mathrm{w})$. Insulin release from the final delivery device was investigated in vitro in media simulating the $\mathrm{pH}$ in rat gastric and small intestinal environments to confirm sufficient capsule coating (Fig. 3). The insulin profile confirmed protection even at a relatively high gastric $\mathrm{pH}$ of 4 , while the exchange of medium to $\mathrm{pH} 7$ initiated the release of insulin, which lasted about $2-3 \mathrm{~h}$.

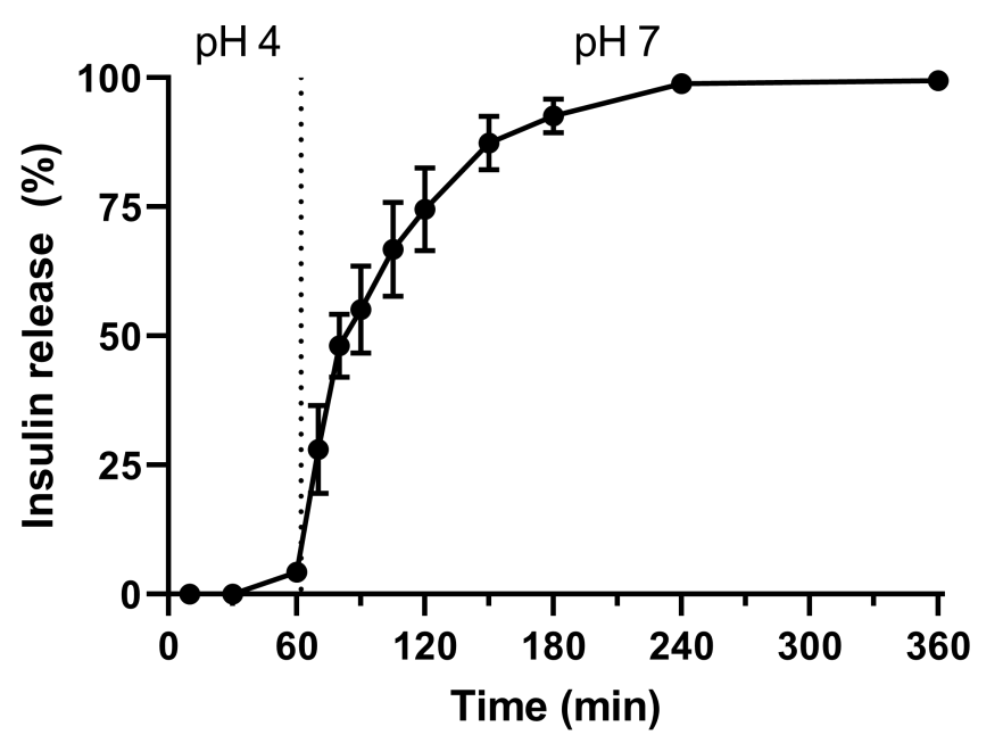

Fig. 3. In vitro release of insulin from the final delivery device at $\mathrm{pH} 4$ followed by $\mathrm{pH} 7$ under sink conditions, mean \pm standard deviation $(n=3)$ - (1-column fitting image).

\subsection{In vivo assessment}

None of the preparation steps towards the final oral delivery device included processes that were likely to compromise the physical stability of insulin, e.g. heat, physical stress, solvents. This was supported by ELISA of diluted in vitro release samples, which confirmed the preservation of the antibody-binding moieties of insulin upon release from the dosage form. An alternative protocol to standard oral gavage was designed for in vivo evaluation of the delivery device, as studies have shown that enteric coated size 9 
capsules may not exit the stomach upon oral administration to rats [18]. The procedure was rendered possible by including a cylindrical magnet in the capsule together with the prepared folded foil (Fig. 4A-B).
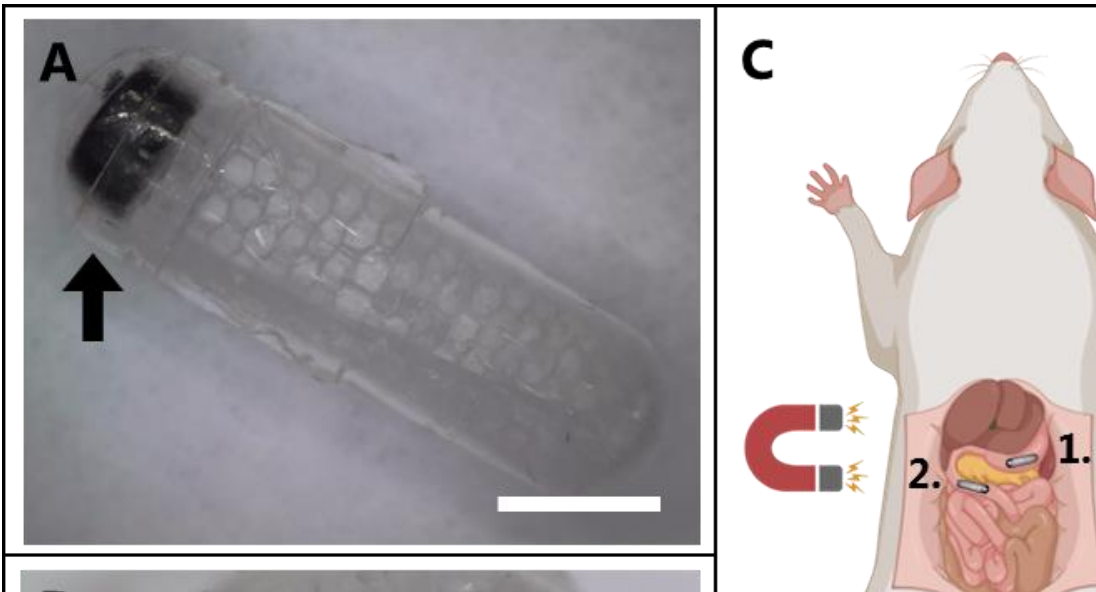

B
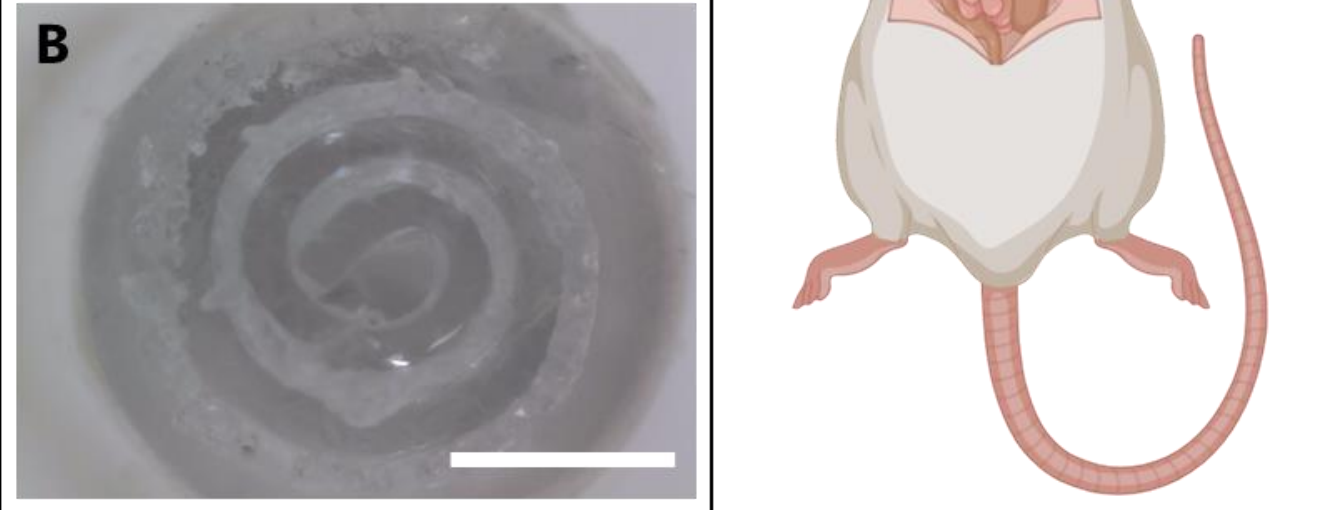

300 Fig. 4. In vivo setup for assessment of the delivery device prototype (A) The delivery device

301 comprised of a Eudragit ${ }^{\circledR}$ L 100-55 coated size 9 gelatin capsule loaded with a magnet (arrow) and

302 a Eudragit $^{\circledR}$ L 100 coated PDMS foil loaded with insulin, sodium dodecyl sulfate and soybean

303 trypsin inhibitor, scale bar: $2 \mathrm{~mm}$ (B) Cross-sectional view of the capsule showing the coiled PDMS

304 foil inside the enteric coated size 9 gelatin capsule, scale bar: $1 \mathrm{~mm}$ (C) Illustration of the in vivo

305 setup in two steps: 1. oral gavage of the delivery device to the stomach, 2. gastric emptying of the

306 delivery device by the use of an external magnet (schematic created with Biorender.com) - (1.5-

307 column fitting image).

308 The delivery device was administered by oral gavage to the stomach of anaesthetized rats

309 followed by duodenal positioning, approximately $3 \mathrm{~cm}$ from the stomach, which was

310 enabled by external magnetic maneuvering of the delivery device through the pylorus (Fig.

$3114 \mathrm{C}$ ). Blood samples were taken over $3 \mathrm{~h}$ after which fully unfolded empty PDMS foils were

312 retrieved from the small intestines (20 $-30 \mathrm{~cm}$ from pylorus) with no residual remains of

313 the gelatin capsules (Fig. 5A). Changes in blood glucose levels were not used for

314 evaluating insulin absorption, as previous data have shown significant blood glucose 
315 lowering effects by anesthesia [23]. Instead, insulin absorption was assessed by plasma 316 human insulin quantification by ELISA, as shown in Fig. 5B.
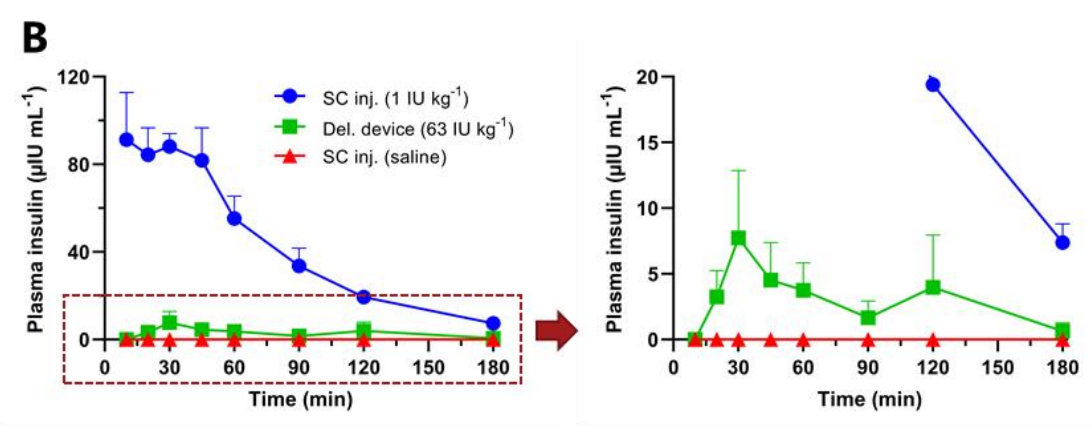

Fig. 5. In vivo performance of the delivery device prototype (A) PDMS foil retrieved after the in vivo study and placed next to a size 9 gelatin capsule for comparison, scale bar: $2 \mathrm{~mm}$ (B) Plasma insulin concentrations in rats as micro international units (IU) per $\mathrm{mL}$ after duodenal insertion of the oral delivery (Del.) device compared to subcutaneous injections (SC inj.) of either insulin or saline shown as mean + standard error of the mean $(n=3-5)$ - (2-column fitting image).

Insulin was present in quantifiable concentrations in the plasma of all five rats receiving the foil-based delivery device, although in relatively low concentrations compared to the SC insulin injections. Additionally, no traces of insulin were detected in any of the negative control plasma samples from rats receiving SC saline injections. The relative oral bioavailability $\left(F_{\text {Rel }}\right)$ of the delivery device compared to the $S C$ insulin injection was found to be $0.12 \pm 0.07 \%$ (mean \pm standard error) based on the respective doses and the area under the curve (AUC) of the plasma insulin profiles, calculated by the following formula.

$$
F_{\text {Rel }}=100 \cdot \frac{A U C_{\text {oral administration }} \cdot 1 \mathrm{IU} \mathrm{kg}}{A U C_{S C \text { injection }} \cdot 63 \mathrm{IU} \mathrm{kg}^{-1}}
$$

Although the relative bioavailability achieved might appear low, oral formulations of the antidiuretic hormone, desmopressin, have previously shown to be both therapeutically and commercially viable with a mean relative bioavailability of $0.1 \%$ compared to SC injection [24]. Moreover, commonly investigated peptides for oral delivery, e.g. desmopressin, octreotide and leuprolide, might comprise a smaller absorption challenge compared to insulin due to its larger size (51 amino acids), two-chain structure and higher susceptibility to proteolysis $[1,12,25]$. However, whether higher bioavailability of such simpler peptides could be achieved by the delivery device remains uncertain at this stage. Other oral delivery strategies have previously resulted in a markedly higher oral insulin bioavailability of $>10 \%$, with the highest numbers being based on physical perforation of the mucosa by 
341 Gl-injecting delivery devices [4-7]. Another study based on local release of thiolated

342 polycarbophil as PE together with insulin from mucoadhesive patches has previously

343 shown a relative oral bioavailability of $2.2 \%$ [26], suggesting that greater absorption by the

344 present unfolding foil might be achievable by incorporating alternative PEs or by

345 improvement of the design of the device itself. The present oral delivery principle could

346 therefore be of general interest to both material scientists and also researchers in the field

347 of oral delivery of peptides and even some small molecule drugs with low oral

348 bioavailability. Class III drug compounds in the Biopharmaceutics Classification System

349 are defined as having high solubility, but low permeability and could possibly benefit from

350 the delivery principle of the foils by creating a local environment of high drug concentration

351 and thus a steep gradient across the intestinal absorptive barrier [27]. However, since the

352 current results are based on an early prototype of utilizing self-unfolding foils, further in-

353 depth studies are needed to investigate the true potential of the delivery concept.

354 Moreover, while the elastomer, PDMS, proved advantageous for achieving self-unfolding

355 properties of the foil, the material possesses the notable disadvantage of not being

356 biodegradable. While PDMS is already used for medical prosthetics and plastic surgery

357 and generally considered nontoxic [28], its lack of disintegration represents a possible risk

358 of accumulation in the Gl-tract upon repeated oral administration. Hence biodegradability

359 would be a considerable advantage for proceeding further from preclinical studies, yet a

360 challenge lies in the creation of a biodegradable elastomeric foil with similar properties.

361 Several approaches towards such materials have previously been investigated for

362 biomedical applications based on alginate, poly(glycerol-sebacate) and even combinations

363 of PDMS and starch [28-31]. For the purpose of oral peptide delivery, it might furthermore

364 be of specific interest to investigate different cavity structures for drug loading and the

365 unfolding forces of different elastomers. The interplay between those aspects might aid

366 perforation through the intestinal mucus thus further decreasing the distance between the

367 point of peptide- and excipient release and the absorptive barrier.

\section{Conclusion}

A new oral delivery concept comprising an elastomeric PDMS foil in an enteric coated gelatin capsule was designed and tested both in vitro and in vivo. The in vitro studies

371 confirmed protection at gastric $\mathrm{pH}$ and fully unfolding properties of the PDMS foil upon 372 capsule disintegration. A new in vivo assessment for enteric coated capsules was applied 373 to ensure gastric emptying of the designed delivery device, which showed promising 374 properties for oral delivery of macromolecules as all rats had quantifiable insulin plasma 
concentrations despite insulin being one of the more challenging peptides to deliver orally. In general, drug compounds with low oral bioavailability, due to low permeation and/or stability, could benefit from confinement in and subsequent release from the foil $[27,32]$. Excipient-driven absorption mechanisms of macromolecules might furthermore gain increased efficiency from co-localization, leading to a reduced requirement of drug. Thus, the concept might have the potential to serve as a platform for a range of drug compounds, yet further studies is needed to fully unravel its potential.

\section{Acknowledgements}

This work was supported by the Danish National Research Foundation (DNRF122) and Villum Foundation (Grant No. 9301), Center for intelligent drug delivery and sensing using microcontainers and nanomechanics (IDUN) and performed in part at DTU Nanolab, the National Centre for Nano Fabrication and Characterization at the Technical University of Denmark. The graphical abstract was created with Biorender.com.

\section{Declaration of interest}

JRJ, LHET, AB, TR and AM have filed a patent application to the European Patent Office on the design of the delivery device.

\section{References}

[1] E. Moroz, S. Matoori, J.-C. Leroux, Oral delivery of macromolecular drugs: Where we are after almost 100 years of attempts, Adv. Drug Deliv. Rev. 101 (2016) 108-121. https://doi.org/10.1016/j.addr.2016.01.010.

[2] S. Maher, B. Ryan, A. Duffy, D.J. Brayden, Formulation strategies to improve oral peptide delivery, Pharm. Pat. Anal. 3 (2014) 313-336. https://doi.org/10.4155/ppa.14.15.

[3] A.N. Zelikin, C. Ehrhardt, A.M. Healy, Materials and methods for delivery of biological drugs, Nat. Chem. 8 (2016) 997-1007. https://doi.org/10.1038/nchem.2629.

[4] A. Abramson, E. Caffarel-Salvador, M. Khang, D. Dellal, D. Silverstein, Y. Gao, M.R. Frederiksen, A. Vegge, F. Hubálek, J.J. Water, A.V. Friderichsen, J. Fels, R.K. Kirk, C. Cleveland, J. Collins, S. Tamang, A. Hayward, T. Landh, S.T. Buckley, N. Roxhed, U. Rahbek, R. Langer, G. Traverso, An ingestible self-orienting system for oral delivery of macromolecules, Science. 363 (2019) 611-615. https://doi.org/10.1126/science.aau2277.

[5] A. Abramson, E. Caffarel-Salvador, V. Soares, D. Minahan, R.Y. Tian, X. Lu, D. Dellal, Y. Gao, S. Kim, J. Wainer, J. Collins, S. Tamang, A. Hayward, T. Yoshitake, H.-C. Lee, J. Fujimoto, J. Fels, M.R. Frederiksen, U. Rahbek, N. Roxhed, R. Langer, G. Traverso, 
A luminal unfolding microneedle injector for oral delivery of macromolecules, Nat. Med. 25 (2019) 1512-1518. https://doi.org/10.1038/s41591-019-0598-9.

[6] G. Traverso, C.M. Schoellhammer, A. Schroeder, R. Maa, G.Y. Lauwers, B.E. Polat, D.G. Anderson, D. Blankschtein, R. Langer, Microneedles for drug delivery via the gastrointestinal tract, J. Pharm. Sci. 104 (2015) 362-367. https://doi.org/10.1002/jps.24182.

[7] M. Hashim, R. Korupolu, B. Syed, K. Horlen, S. Beraki, P. Karamchedu, A.K. Dhalla, R. Ruffy, M. Imran, Jejunal wall delivery of insulin via an ingestible capsule in anesthetized swine-A pharmacokinetic and pharmacodynamic study, Pharmacol. Res. Perspect. 7 (2019) e00522. https://doi.org/10.1002/prp2.522.

[8] S. Maher, D.J. Brayden, L. Casettari, L. Illum, Application of Permeation Enhancers in Oral Delivery of Macromolecules: An Update, Pharmaceutics. 11 (2019) 41. https://doi.org/10.3390/pharmaceutics11010041.

[9] F. McCartney, J.P. Gleeson, D.J. Brayden, Safety concerns over the use of intestinal permeation enhancers: A mini-review, Tissue Barriers. 4 (2016) e1176822. https://doi.org/10.1080/21688370.2016.1176822.

[10] N. Barker, Adult intestinal stem cells: critical drivers of epithelial homeostasis and regeneration, Nat. Rev. Mol. Cell Biol. 15 (2014) 19-33. https://doi.org/10.1038/nrm3721.

[11] I.B. Halberg, K. Lyby, K. Wassermann, T. Heise, E. Zijlstra, L. Plum-Mörschel, Efficacy and safety of oral basal insulin versus subcutaneous insulin glargine in type 2 diabetes: a randomised, double-blind, phase 2 trial, Lancet Diabetes Endocrinol. 7 (2019) 179-188. https://doi.org/10.1016/S2213-8587(18)30372-3.

[12] S.T. Buckley, T.A. Bækdal, A. Vegge, S.J. Maarbjerg, C. Pyke, J. Ahnfelt-Rønne, K.G. Madsen, S.G. Schéele, T. Alanentalo, R.K. Kirk, B.L. Pedersen, R.B. Skyggebjerg, A.J. Benie, H.M. Strauss, P.-O. Wahlund, S. Bjerregaard, E. Farkas, C. Fekete, F.L. Søndergaard, J. Borregaard, M.-L. Hartoft-Nielsen, L.B. Knudsen, Transcellular stomach absorption of a derivatized glucagon-like peptide-1 receptor agonist, Sci. Transl. Med. 10 (2018) eaar7047. https://doi.org/10.1126/scitranslmed.aar7047.

[13] A. Banerjee, J. Lee, S. Mitragotri, Intestinal mucoadhesive devices for oral delivery of insulin, Bioeng. Transl. Med. 1 (2016) 338-346. https://doi.org/10.1002/btm2.10015.

[14] K. Whitehead, Z. Shen, S. Mitragotri, Oral delivery of macromolecules using intestinal patches: applications for insulin delivery, J. Control. Release. 98 (2004) 3745. https://doi.org/10.1016/j.jconrel.2004.04.013.

[15] A. Ahmed, C. Bonner, T.A. Desai, Bioadhesive microdevices with multiple reservoirs: a new platform for oral drug delivery, J. Control. Release. 81 (2002) 291306. https://doi.org/10.1016/S0168-3659(02)00074-3.

[16] J.R. Jørgensen, M.L. Jepsen, L.H. Nielsen, M. Dufva, H.M. Nielsen, T. Rades, A. Boisen, A. Müllertz, Microcontainers for oral insulin delivery - In vitro studies of permeation enhancement, Eur. J. Pharm. Biopharm. 143 (2019) 98-105. https://doi.org/10.1016/j.ejpb.2019.08.011. 
[17] J.R. Jørgensen, F. Yu, R. Venkatasubramanian, L.H. Nielsen, H.M. Nielsen, A. Boisen, T. Rades, A. Müllertz, In vitro, ex vivo and in vivo evaluation of microcontainers for oral delivery of insulin, Pharmaceutics. 12 (2020) 48. https://doi.org/10.3390/pharmaceutics12010048.

[18] S. Saphier, A. Rosner, R. Brandeis, Y. Karton, Gastro intestinal tracking and gastric emptying of solid dosage forms in rats using X-ray imagining, Int. J. Pharm. 388 (2010) 190-195. https://doi.org/10.1016/j.ijpharm.2010.01.001.

[19] A. Banerjee, K. Ibsen, T. Brown, R. Chen, C. Agatemor, S. Mitragotri, Ionic liquids for oral insulin delivery, PNAS. 115 (2018) 7296-7301. https://doi.org/10.1073/pnas.1722338115.

[20] S.K. Srivastava, F. Ajalloueian, A. Boisen, Thread-Like Radical-Polymerization via Autonomously Propelled (TRAP) Bots, Adv. Mater. 31 (2019) 1901573. https://doi.org/10.1002/adma.201901573.

[21] L.H. Nielsen, S.S. Keller, A. Boisen, Microfabricated devices for oral drug delivery, Lab. Chip. 18 (2018) 2348-2358. https://doi.org/10.1039/C8LC00408K.

[22] K. Vdoviaková, E. Petrovová, M. Maloveská, L. Krešáková, J. Teleky, M.Z.J. Elias, D. Petrášová, Surgical anatomy of the gastrointestinal tract and its vasculature in the laboratory rat, Gastroenterol. Res. Pract. 2016 (2016) 2632368. https://doi.org/10.1155/2016/2632368.

[23] S. Harloff-Helleberg, L.H. Nielsen, H.M. Nielsen, Animal models for evaluation of oral delivery of biopharmaceuticals, J. Control. Release. 268 (2017) 57-71. https://doi.org/10.1016/j.jconrel.2017.09.025.

[24] A. Fjellestad-Paulsen, P. Höglund, S. Lundin, O. Paulsen, Pharmacokinetics of 1deamino-8-d-arginine vasopressin after various routes of administration in healthy volunteers, Clin. Endocrinol. (Oxf.). 38 (1993) 177-182. https://doi.org/10.1111/j.13652265.1993.tb00990.x.

[25] J. Wang, V. Yadav, A.L. Smart, S. Tajiri, A.W. Basit, Toward oral delivery of biopharmaceuticals: An assessment of the gastrointestinal stability of 17 peptide drugs, Mol. Pharm. 12 (2015) 966-973. https://doi.org/10.1021/mp500809f.

[26] V. Grabovac, F. Föger, A. Bernkop-Schnürch, Design and in vivo evaluation of a patch delivery system for insulin based on thiolated polymers, Int. J. Pharm. 348 (2008) 169-174. https://doi.org/10.1016/j.ijpharm.2007.06.052.

[27] T. Flanagan, Potential for pharmaceutical excipients to impact absorption: A mechanistic review for BCS Class 1 and 3 drugs, Eur. J. Pharm. Biopharm. 141 (2019) 130-138. https://doi.org/10.1016/j.ejpb.2019.05.020.

[28] L. Ceseracciu, J.A. Heredia-Guerrero, S. Dante, A. Athanassiou, I.S. Bayer, Robust and biodegradable elastomers based on corn starch and polydimethylsiloxane (PDMS), ACS Appl. Mater. Interfaces. 7 (2015) 3742-3753. https://doi.org/10.1021/am508515z.

[29] H. Daemi, S. Rajabi-Zeleti, H. Sardon, M. Barikani, A. Khademhosseini, H. Baharvand, A robust super-tough biodegradable elastomer engineered by 
supramolecular ionic interactions, Biomaterials. 84 (2016) 54-63. https://doi.org/10.1016/j.biomaterials.2016.01.025.

494 [30] Y. Wang, G.A. Ameer, B.J. Sheppard, R. Langer, A tough biodegradable elastomer, 495 Nat. Biotechnol. 20 (2002) 602-606. https://doi.org/10.1038/nbt0602-602.

[31] B.G. Amsden, Biodegradable elastomers in drug delivery, Expert Opin. Drug Deliv. 5 (2008) 175-187. https://doi.org/10.1517/17425247.5.2.175.

[32] L.H. Nielsen, S.S. Keller, K.C. Gordon, A. Boisen, T. Rades, A. Müllertz, Spatial confinement can lead to increased stability of amorphous indomethacin, Eur. J. Pharm. Biopharm. 81 (2012) 418-425. https://doi.org/10.1016/j.ejpb.2012.03.017. 